\title{
Comparative Study of Vibrational Spectra of Two Bioactive Natural Products Lupeol and Lupenone Using MM/QM Method
}

\author{
Apoorva Dwivedi, ${ }^{1}$ Anoop Kumar Pandey, ${ }^{1}$ Kanwal Raj, ${ }^{2}$ and Neeraj Misra ${ }^{1}$ \\ ${ }^{1}$ Department of Physics, Lucknow University, Lucknow 226007, India \\ ${ }^{2}$ Central Drug Research Institute, Lucknow 226007, India
}

Correspondence should be addressed to Kanwal Raj, kanwalraj12@ rediffmail.com and

Neeraj Misra, neerajmisra11@gmail.com

Copyright (C) 2012 Apoorva Dwivedi et al. This is an open access article distributed under the Creative Commons Attribution License, which permits unrestricted use, distribution, and reproduction in any medium, provided the original work is properly cited.

\begin{abstract}
This work deals with the theoretical study on the molecular structure and vibrational spectra of two well-known natural products: lupeol and lupenone. The spectra were interpreted with the aid of normal mode analysis following fullstructure optimization carried out with the hybrid two-level ONIOM (B3LYP/6-31G: PM3) method. A detailed interpretation of the infrared spectra of Lupeol and Lupenone is also reported in the present work. The similarities and differences between the vibrational spectra of the two molecules studied have been highlighted. The scaled theoretical wave numbers are in perfect agreement with the experimental values. The thermodynamic calculations related to the title compounds were also performed at B3LYP/6-31G: PM3 level of theory. Quantum chemical calculations have been carried out to understand the dynamical behavior of the bioactive molecules Lupeol and Lupenone.
\end{abstract}

Keywords: Molecular structure, Vibrational spectra, FTIR and FT-Raman spectra, Oniom, Lupeol and Lupenone

\section{Introduction}

Crataeva nurvala (Capparidaceae) is a plant from which Lupeol has been isolated, and its reported antimalarial activity prompted to prepare structurally modified analogues for the development of new antimalarial agent for better activity. In the treatment of malaria, natural products are an unending source of new lead, and their role is not new. The structure of Lupeol is reminiscent of that of cholesterol, and the compound is expected to be able to enter the cellular membranes. Due to the presence of a single hydroxy group and a large, apolar skeleton, Lupeol acts as an amphiphile. According to the bilayer hypothesis, stomatocytes are generally formed when a lipophilic compound is incorporated and eventually expands the inner layer of the lipid membrane. Such changes appear to be more prohibiting with respect to parasite growth than the incorporation of an amphiphile into the outer layer, as in case of echinocytogenic compounds. The two possible sites for chemical modification present in Lupeol are ring A, and isopropenyl side chain. Modification on ring A includes, ring-A expansion and cleavage are followed 


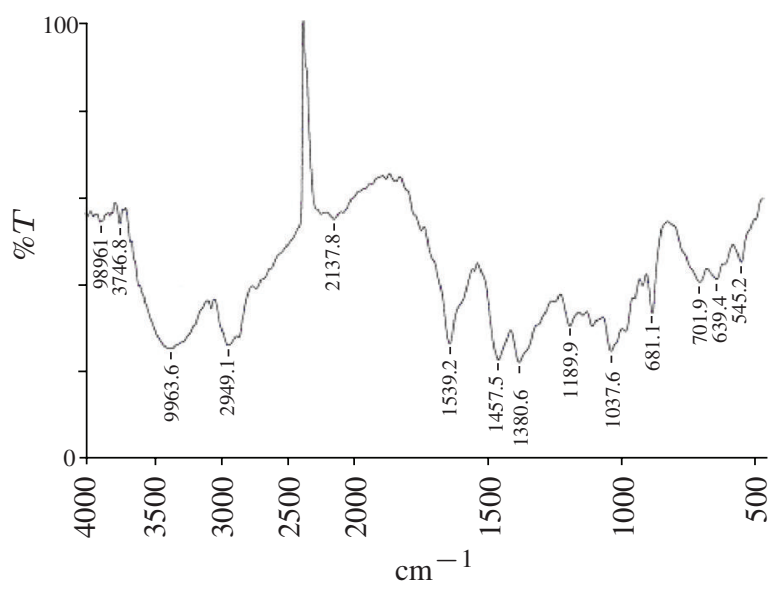

Figure 1: FTIR spectra of lupeol.

by introduction of antimalarial pharmacophores and modification at isopropenyl side chain [1-4]. Lupeol is a naturally occurring triterpene isolated from Crataeva nurvala (Varuna) stem bark. Naturally occurring pentacyclic triterpenes of plant origin have a wide range of biological effects that render them useful in cosmeceutical and nutraceutical applications. In Ayurveda, C. nurvala is recommended in the management of urinary disorders. Pharmacological studies using the root bark and stem bark of $C$. nurvala have confirmed its beneficial effects in urinary disorders and revealed its potential benefits in the management of arthritic conditions [5]. In another recent study, lupeol and lupeol linoleate investigated for their anti-inflammatory, antinociceptive, antipyretic, and ulcerogenic properties in comparison with the commonly used nonsteroidal anti-inflammatory drug, indomethacin in rats. Lupeol, lupeol linoleate, and indomethacin showed a reduction in paw swelling by 39,58, and 35\%, respectively, in adjuvant arthritis. Lupeol and lupeol linoleate were devoid of any antinociceptive, antipyretic, and ulcerogenic actions unlike indomethacin suggesting that the mechanism of action of triterpenes is different from the nonsteroidal anti-inflammatory drug [6].

\section{Experimental and Computational Detail}

Fourier transform infrared spectra were recorded with FT-IR Perkin Elmer spectrometer in $\mathrm{KBr}$ dispersion in the range of 500 to $4000 \mathrm{~cm}^{-1}$ for both the molecules. The experimental FT-IR spectra for lupeol and lupenone are given in Figures 1 and 2, respectively. All the calculations were performed by using the hybrid two-level Oniom (B3LYP/6-31G: PM3) method using the GAUSSIAN 09 suite of program [7]. In order to avoid time-consuming calculations for large systems in following three ways: (i) using cheap molecular mechanics or semiempirical methods to study large systems $[8,9]$, (ii) using the periodic boundary conditions to mimic the large periodic systems [10,11], (iii) using the more accurate quantum chemistry methods to study small model molecules designed to mimic the behavior of large real systems [12-14]. The emerging hybrid quantum chemical/molecular mechanical method, ONIOM, incorporating the advantages of both approaches has been set up recently. It has been used extensively for the calculations of macromolecules in biosystems [15-19]. ONIOM divides the system into three 


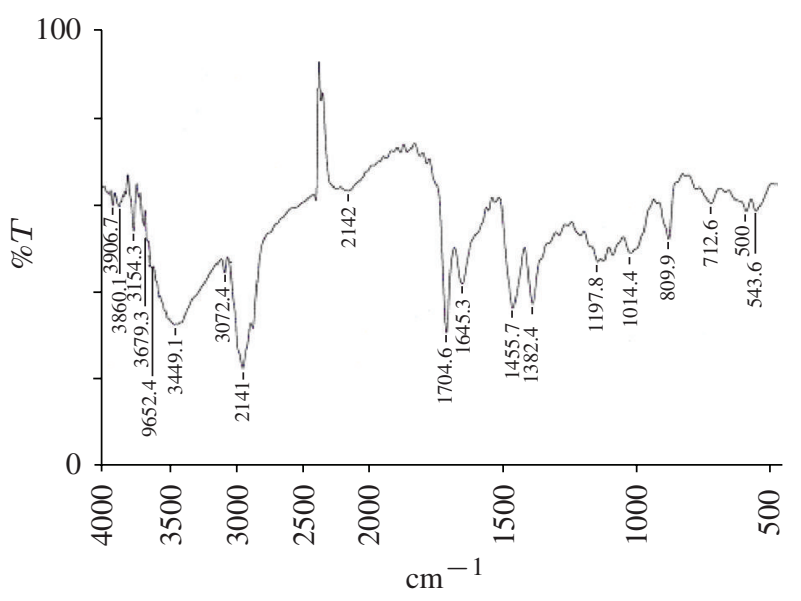

Figure 2: FTIR spectra of lupenone.

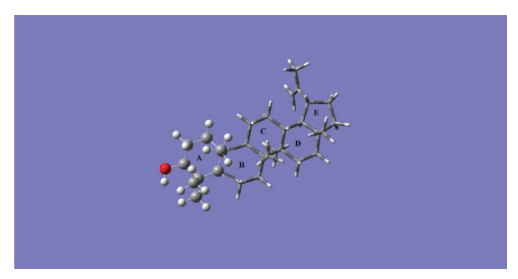

Figure 3: Model molecular structure of lupeol.

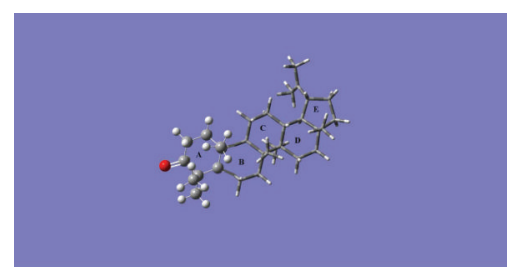

Figure 4: Model molecular structure of lupenone.

segments which can deal with the complicated calculations at different levels. A major advantage of the ONIOM method is the speed of computation compared to full DFT calculations. In these molecules, atoms in ring A and its adjacent atoms are treated quantum mechanically (B3LYP/DFT), and these atoms are shown by balls and sticks in Figures 3 and 4, while the remaining part of the molecule is treated by semiempirical PM3 method.

\section{Result and Discussion}

Theoretical calculations for lupeol and lupenone were carried out using the ONIOM (B3LYP/6-31G: PM3) method. The optimized structure parameters of lupeol and lupenone are listed in Table 1. By 
Table 1: Calculated bond lengths (angstroms) and bond angle (degrees) for lupeol and lupenone, which were optimized at the Oniom (B3LYP/6-31G: PM3) methods.

\begin{tabular}{|c|c|}
\hline \multicolumn{2}{|c|}{ Lupeol } \\
\hline Bond lengths & Oniom (B3LYP/6-31G: PM3) \\
\hline$(\mathrm{C}-\mathrm{C})$ & $1.487-1.577$ \\
\hline$(\mathrm{C}=\mathrm{C})$ & 1.333 \\
\hline$(\mathrm{C}-\mathrm{H})$ & $1.085-1.133$ \\
\hline$(\mathrm{C}-\mathrm{O})$ & 1.415 \\
\hline$(\mathrm{O}-\mathrm{H})$ & 0.952 \\
\hline Bond angles & Oniom (B3LYP/6-31G: PM3) \\
\hline$(\mathrm{C}-\mathrm{C}-\mathrm{C})$ & $101.77-115.51$ \\
\hline$(\mathrm{C}-\mathrm{C}=\mathrm{C})$ & $121.97-122.51$ \\
\hline$(\mathrm{C}-\mathrm{C}-\mathrm{H})$ & $107.11-112.12$ \\
\hline$(\mathrm{C}-\mathrm{O}-\mathrm{H})$ & 106.02 \\
\hline$(\mathrm{C}-\mathrm{C}-\mathrm{O})$ & $107.61-113.37$ \\
\hline$(\mathrm{C}=\mathrm{C}-\mathrm{H})$ & $121.22-123.46$ \\
\hline$(\mathrm{H}-\mathrm{C}-\mathrm{H})$ & $105.341-115.30$ \\
\hline \multicolumn{2}{|c|}{ Lupenone } \\
\hline Bond lengths & Oniom (B3LYP/6-31G: PM3) \\
\hline$(\mathrm{C}-\mathrm{C})$ & $1.502-1.580$ \\
\hline$(\mathrm{C}-\mathrm{H})$ & $1.098-1.121$ \\
\hline$(\mathrm{C}=\mathrm{O})$ & 1.215 \\
\hline$(\mathrm{C}=\mathrm{C})$ & 1.345 \\
\hline Bond angles & Oniom (B3LYP/6-31G: PM3) \\
\hline$(\mathrm{C}-\mathrm{C}-\mathrm{C})$ & $101.46-125.97$ \\
\hline$(\mathrm{C}-\mathrm{C}-\mathrm{H})$ & $104.15-112.67$ \\
\hline$(\mathrm{C}-\mathrm{C}=\mathrm{O})$ & $120.92-122.47$ \\
\hline$(\mathrm{H}-\mathrm{C}-\mathrm{H})$ & $104.52-107.26$ \\
\hline$(\mathrm{C}=\mathrm{C}-\mathrm{C})$ & $120.48-130.21$ \\
\hline
\end{tabular}

allowing the relaxation of all parameters, the calculations converge to optimized geometries, which correspond to true energy minima, as revealed by the lack of imaginary frequencies in the vibrational mode calculation. Subsequently, the global minimum energy obtained for structure optimization of lupeol and lupenone were found out to be -0.1855 a.u and -0.1666 a.u., respectively. The energy difference between the two compounds was calculated to be 0.0189 a.u. Thermodynamic properties of the lupeol and lupenone were calculated by B3LYP/6-31G: PM3 levels and are given in Table 2.

\subsection{Molecular Geometry Optimization}

In case of lupeol, the molecule has five rings. Out of these four are six membered and one five membered. The optimized bond length of $\mathrm{C}-\mathrm{C}$ in six-membered cyclohexane ring $\mathrm{A}$ ranges between $1.518 \AA$ and $1.561 \AA$, while for another cyclohexane ring $\mathrm{B}$ this ranges between $1.512 \AA$ and $1.557 \AA$. The optimized 
Table 2: Calculated thermodynamic properties of lupeol and lupenone by oniom (B3LYP/6-31G: PM3) methods.

\begin{tabular}{lcccccc}
\hline & \multicolumn{2}{c}{$\mathrm{E}(\mathrm{thermal})\left(\mathrm{kcalmol}^{-1}\right)$} & \multicolumn{2}{c}{$\mathrm{CV}\left(\mathrm{calK}^{-1} \mathrm{~mol}^{-1}\right)$} & \multicolumn{2}{c}{$\mathrm{S}\left(\mathrm{calK}^{-1} \mathrm{~mol}^{-1}\right)$} \\
& Lupeol & Lupenone & Lupeol & Lupenone & Lupeol & Lupenone \\
\hline Total & 485.297 & 472.295 & 126.014 & 123.087 & 177.103 & 173.994 \\
Translational & 0.889 & 0.889 & 2.981 & 2.981 & 44.041 & 44.027 \\
Rotational & 0.889 & 0.889 & 2.981 & 2.981 & 36.352 & 36.339 \\
Vibrational & 483.519 & 470.517 & 120.053 & 117.126 & 96.710 & 93.628 \\
\hline
\end{tabular}

bond length of $\mathrm{C}-\mathrm{C}$ in six-membered cyclohexane ring $\mathrm{C}$ ranges between $1.518 \AA$ and $1.572 \AA$, while for another cyclohexane ring $\mathrm{D}$ this ranges between $1.525 \AA$ and $1.547 \AA$. For five-membered ring $\mathrm{E}$ (cyclopentane), $\mathrm{C}-\mathrm{C}$ bond lengths are quite high and ranges between $1.534 \AA$ and $1.548 \AA$. The optimized value of $\mathrm{C}-\mathrm{C}$ bond length adjacent to ring $\mathrm{E}$ is found to be $1.481-1.502 \AA$, which is lower in comparison to the $\mathrm{C}-\mathrm{C}$ bond length in other rings The optimized $\mathrm{C}-\mathrm{O}$ bond length adjacent to ring $\mathrm{A}$ is found to be $1.415 \AA$, and the optimized $\mathrm{O}-\mathrm{H}$ bond length adjacent to ring $\mathrm{A}$ is found to be $0.952 \AA$.

For lupenone, the given molecule has five rings. Out of these four are six membered and one five membered. The optimized bond length of $\mathrm{C}-\mathrm{C}$ in six membered cyclohexane ring $\mathrm{A}$ ranges between $1.515 \AA$ and $1.550 \AA$, while for another cyclohexane ring $\mathrm{B}$ this ranges between $1.519 \AA$ and $1.555 \AA$. The optimized bond length of $\mathrm{C}-\mathrm{C}$ in six-membered cyclohexane ring $\mathrm{C}$ ranges between $1.517 \AA$ and $1.580 \AA$, while for another cyclohexane ring $\mathrm{D}$ this ranges between $1.526 \AA$ and $1.565 \AA$. For five membered (cyclopentane) ring E, C-C bond lengths range between $1.507 \AA$ and $1.565 \AA$. The optimized value of $\mathrm{C}-\mathrm{C}$ bond length adjacent to ring $\mathrm{E}$ is found to be $1.484-1.492 \AA$, which is lower in comparison to the $\mathrm{C}-\mathrm{C}$ bond length in other rings. The optimized carbonyl group $\mathrm{C}=\mathrm{O}$ bond length adjacent to ring $\mathrm{A}$ is found to be $1.215 \AA$, and the optimized $\mathrm{C}=\mathrm{C}$ bond length adjacent to ring $\mathrm{A}$ is found to be $1.345 \AA$. This difference in the $\mathrm{C}-\mathrm{C}$ bond length is attributed to the difference in bond strength. The length of a chemical bond is the result of equilibrium of attractive and repulsive forces between the atoms which are bonded. In fact, the distance between the atoms of a bond is not constant since the atoms are always vibrating in a molecules; the measured bond distance is therefore an average value. Although the measurements may vary in accuracy, but still similar bonds have fairly constant lengths in different molecules, variation generally being very less.

\subsection{Vibrational Assignments}

The molecules, lupeol and lupenone contain, 81 and 79 atoms, and they have 237 and 231 normal modes of vibrations, respectively. All the fundamental vibrations are active IR. The harmonic-vibrational frequencies are calculated for both the molecules at B3LYP/6-31G: PM3 level, and experimental frequencies (FTIRs) have been compared in Tables 3 and 4 for lupeol and lupenone, respectively. Tables 3 and 4 also include the vibrational assignments of the normal modes. Vibrational assignments are based on the observation of the animated modes in GaussView [20] and assignments reported in literature.

In lupeol, the $\mathrm{O}-\mathrm{H}$ functional group is present at $3721 \mathrm{~cm}^{-1}$ in calculated spectra which is in good agreement with the experimental data. The $\mathrm{C}-\mathrm{H}$ functional group is present at a number of positions. The 
Table 3: Comparison of the observed and calculated vibrational spectra of lupeol with (B3LYP/6-31G: PM3) methods.

\begin{tabular}{|c|c|c|c|}
\hline $\begin{array}{l}\text { B3LYP } \\
\text { (Calc) }\end{array}$ & IR (Int.) & IR (Exp) & Vibrational assignments [P.E.D.] \\
\hline 259 & 8.573 & - & $\operatorname{Rock}\left(\mathrm{C}-\mathrm{CH}_{3}\right)$ \\
\hline 317 & 34.472 & - & $\omega(\mathrm{O}-\mathrm{H})$ \\
\hline 556 & 5.331 & 546 & $\tau(\mathrm{C}-\mathrm{C}-\mathrm{C}=\mathrm{C})$ \\
\hline 941 & 8.306 & - & $\beta(\mathrm{C}-\mathrm{C}-\mathrm{H})$ \\
\hline 952 & 9.997 & $950(\mathrm{sh})$ & $\operatorname{Rock}\left(\mathrm{C}=\mathrm{CH}_{2}\right)+\operatorname{Rock}\left(\mathrm{C}-\mathrm{CH}_{3}\right)$ \\
\hline 999 & 14.419 & - & Rock $\mathrm{CH}_{2}$ \\
\hline 1007 & 13.866 & $1005(\mathrm{sh})$ & Twist $\mathrm{CH}_{3}$ \\
\hline 1089 & 5.420 & 1037 & Twist $\mathrm{CH}_{2}$ \\
\hline 1101 & 8.428 & - & Rock $\mathrm{CH}_{2}$ \\
\hline 1137 & 4.075 & $1145(\mathrm{sh})$ & $\beta(\mathrm{C}-\mathrm{C}-\mathrm{H})$ \\
\hline 1229 & 10.558 & 1189 & $\beta(\mathrm{C}-\mathrm{C}-\mathrm{H})$ \\
\hline 1292 & 7.878 & $1290(\mathrm{sh})$ & $\beta(\mathrm{C}-\mathrm{C}-\mathrm{H})$ \\
\hline 1328 & 20.581 & - & $\beta(\mathrm{C}-\mathrm{C}-\mathrm{H})$ \\
\hline 1360 & 4.138 & - & $\beta(\mathrm{H}-\mathrm{C}-\mathrm{H})$ \\
\hline 1398 & 11.404 & 1380 & $\beta(\mathrm{C}-\mathrm{O}-\mathrm{H})+\beta(\mathrm{C}-\mathrm{C}-\mathrm{H})$ \\
\hline 2935 & 5.811 & 2946 & $\nu(\mathrm{C}-\mathrm{H})$ \\
\hline 2991 & 8.277 & - & $\nu(\mathrm{C}-\mathrm{H})$ \\
\hline 3051 & 10.361 & $3051(\mathrm{sh})$ & $\nu(\mathrm{C}-\mathrm{H})$ \\
\hline 3721 & 12.146 & 3746 & $\nu(\mathrm{O}-\mathrm{H})$ \\
\hline
\end{tabular}

Abbreviations: $\nu$ : stretching; $\beta$ : bending; S: scissoring; w: wagging; $\tau$ : torsion; sh: shoulder.

stretching vibration, $\nu(\mathrm{C}-\mathrm{H})$, is expected to occur in the region $2800-3200 \mathrm{~cm}^{-1}$. The $\nu(\mathrm{C}-\mathrm{H})$ functional group is present at $2935 \mathrm{~cm}^{-1}$ in calculated spectra which is in good agreement with the experimental data, that is, $2946 \mathrm{~cm}^{-1}$. The calculated values of the $\nu(\mathrm{C}-\mathrm{H})$ vibration lie within this spectral range. The calculated (scaled) and experimental frequencies are deviated by large amount, which may be explained by the presence of hydrogen bondings in the solid sample. The theoretical calculations have been done on gas-phase molecule.

The $-\mathrm{CH}_{2}$ and $-\mathrm{CH}_{3}$ functional groups are important constituents of lupeol, and vibrations corresponding to these groups are present in a number of modes. The stretching modes corresponding to these groups are of two types-symmetric and asymmetric stretching's. In the symmetric stretching vibration, both the arms of the functional group are stretching in phase with each other, whereas, in the asymmetric stretching mode, the atomic hydrogen motions are out of phase. The twisting and rocking vibrations for both the functional groups are present mixed with other vibrations in middle region. As expected, the ring torsion modes along with wagging modes appear in the low-frequency range. The wagging vibration $\omega(\mathrm{O}-\mathrm{H})$ is present at $317 \mathrm{~cm}^{-1}$ in calculated spectra in lower region, and an intense band due to torsion of $\mathrm{C}-\mathrm{C}-\mathrm{C}=\mathrm{C}$ is at $556 \mathrm{~cm}^{-1}$ in calculated spectrum that matches well with experimental one, that is, $546 \mathrm{~cm}^{-1}$. There are some frequencies in lower region having appreciable IR intensity. Furthermore, 
Table 4: Comparison of the observed and calculated vibrational spectra of lupenone with (B3LYP/631G: PM3) methods.

\begin{tabular}{lccc}
\hline B3LYP (Calc) & IR (Int.) & IR (Exp) & Vibrational assignments [P.E.D.] \\
\hline 602 & 7.414 & 580 & $\beta(\mathrm{C}-\mathrm{C}=\mathrm{O})+\beta(\mathrm{C}-\mathrm{C}-\mathrm{C})$ \\
793 & 3.127 & 712 & $\beta(\mathrm{C}-\mathrm{C}-\mathrm{H})$ \\
952 & 5.632 & - & Rock $\left(\mathrm{C}-\mathrm{CH}_{2}\right)+$ Rock $\left(\mathrm{C}-\mathrm{CH}_{3}\right)$ \\
962 & 4.246 & - & Rock CH$_{2}$ \\
1005 & 18.283 & 1014 & $\beta(\mathrm{C}-\mathrm{C}-\mathrm{H})$ \\
1040 & 7.213 & - & Twist $\mathrm{CH}_{2}$ \\
1056 & 9.850 & $1055(\mathrm{sh})$ & Rock $\mathrm{CH}_{2}$ \\
1123 & 4.486 & - & Twist $\mathrm{CH}_{2}$ \\
1131 & 5.153 & 1137 & Twist CH \\
1152 & 4.690 & $1155(\mathrm{sh})$ & Rock CH \\
1185 & 3.506 & $1180(\mathrm{sh})$ & $\beta(\mathrm{C}-\mathrm{C}-\mathrm{H})$ \\
1255 & 4.951 & - & $\beta(\mathrm{C}-\mathrm{C}-\mathrm{H})$ \\
1289 & 9.791 & $1285(\mathrm{sh})$ & $\beta(\mathrm{C}-\mathrm{C}-\mathrm{H})$ \\
1917 & 234.342 & - & $\nu(\mathrm{C}=\mathrm{O})$ \\
2734 & 4.850 & - & $\nu(\mathrm{C}-\mathrm{H})$ \\
2934 & 4.346 & - & $\nu(\mathrm{C}-\mathrm{H})$ \\
2976 & 2.869 & $\nu(\mathrm{C}-\mathrm{H})$ \\
\hline
\end{tabular}

Abbreviations: $\nu$ : stretching; $\beta$ : bending; S: scissoring; w: wagging; $\tau$ : torsion; sh: shoulder.

the study of low frequency vibrations is of great significance because it gives information on weak intermolecular interactions, which takes place in enzyme reactions [21]. Knowledge of low frequency mode is also essential for the interpretation of the effect of electromagnetic radiation on biological systems [22].

In lupenone, the $\mathrm{C}-\mathrm{H}$ functional group is present at a number of positions. The $\nu(\mathbf{C}-\mathrm{H})$ functional group is present at $2934 \mathrm{~cm}^{-1}$ in calculated spectra which is in good agreement with the experimental data, that is, $2941 \mathrm{~cm}^{-1}$. The calculated values of the $\nu(\mathrm{C}-\mathrm{H})$ vibration lie within this spectral range. The region $1600-2000 \mathrm{~cm}^{-1}$ is generally considered as the double bond stretching region for $\mathrm{C}=\mathrm{O}, \mathrm{C}=\mathrm{C}$, and $\mathrm{C}=\mathrm{N}$ bonds [23-26]. The $\mathrm{C}=\mathrm{O}$ stretching vibration, $\nu(\mathrm{C}=\mathrm{O})$, appears as a prominent mode in the calculated spectra at $1917 \mathrm{~cm}^{-1}$.

The $-\mathrm{CH}_{2}$ and $-\mathrm{CH}_{3}$ functional groups are important constituents of lupeol, and vibrations corresponding to these groups are present in a number of modes. Due to twist $-\mathrm{CH}_{2}$ mode, an intense peak is present at $1131 \mathrm{~cm}^{-1}$ in the calculated spectra which matches well with the experimental one, that is, $1137 \mathrm{~cm}^{-1}$. The twisting and rocking vibrations for both the functional groups are present mixed with other vibrations in the middle region. Here, we are discussing only most IR-active modes present in calculated spectra for both the molecules. The aim of this discussion is to obtain direct information on lower- and higher-frequency vibrations of the two biologically active molecules, that is, lupeol and lupenone. 


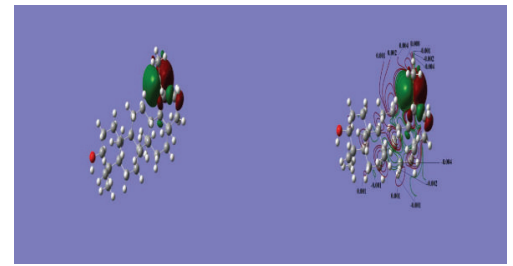

Figure 5: 3D and 2D plots of highest occupied molecular orbital for lupeol.

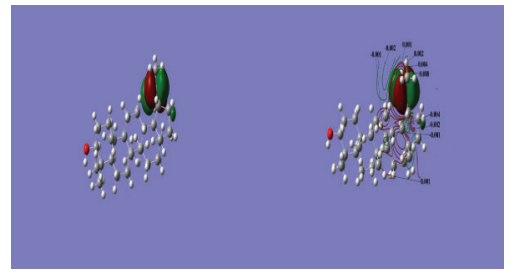

Figure 6: 3D and 2D plots of lowest unoccupied molecular orbital for lupeol.

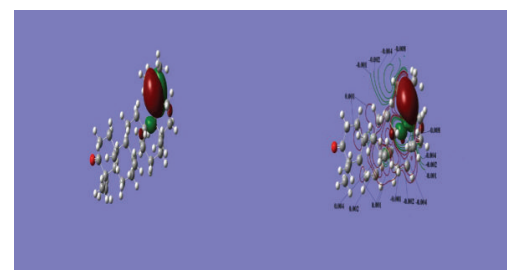

Figure 7: 3D and 2D plots of highest occupied molecular orbital for lupenone.

\subsection{Electronic Properties and Dipole Moment}

The frontier orbitals, HOMO and LUMO determine the way the molecule interacts with other species. The frontier orbital gap helps to characterize the chemical reactivity and kinetic stability of the molecule. A molecule which has a larger orbital gap is more polarized having more reactive part as far as reaction is concerned [27]. According to the present Oniom calculations, the frontier orbital gap in case of the given molecule is 10.09 and $11.15 \mathrm{eV}$, respectively for lupeol and lupenone given in Table 5. The 3D and 2D plots of the HOMO, LUMO and electrostatic potential for both the molecules are shown in Figures 3-7. For lupeol, HOMO is located adjacent to the ring E. If we see 2D plot of HOMO (Figure 5) negative equipotential concentric lines appear near and adjacent to the ring E. However, surfaces of positive lines are closely concentrated over ring A \& B and D \& E. LUMO is located (Figure 6) at the same place as HOMO that is, close to ring E. The electro negative lines are towards the outer part and near to ring $\mathrm{E}$ and positive lines are located nearly over the rings $\mathrm{C}, \mathrm{D}$ and $\mathrm{E}$, respectively.

For lupenone, HOMO is located adjacent to the ring E. If we see 2D plot of HOMO (Figure 7) negative equipotential concentric lines appears near and adjacent to the ring $\mathrm{E}$ and $\mathrm{D}$. However, contours of positive lines are closely concentrated over and adjacent to ring A, B and C. LUMO is located (Figure 8) at a different place near and over the ring A. The electro negative lines are towards the outer and inside parts of ring $\mathrm{A}, \mathrm{B}$ and $\mathrm{C}$ and positive lines are located at the lower part of the ring $\mathrm{A}$ and B. 


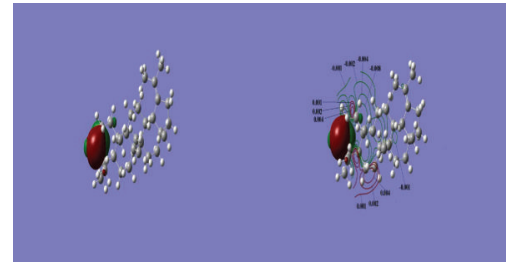

Figure 8: 3D and 2D plots of lowest unoccupied molecular orbital for lupenone.

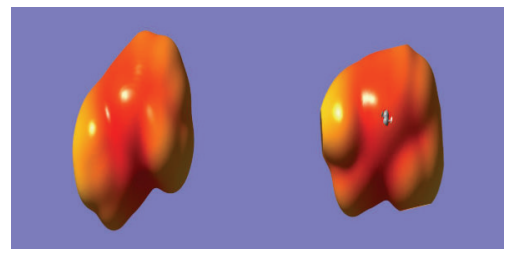

Figure 9: Molecular electrostatic potential plots of lupeol and lupenone.

Table 5: Lowest energy, HOMO-LUMO gap (frontier orbital energy gap) and dipole moment of lupeol and lupenone by (B3LYP/6-31G: PM3) methods.

\begin{tabular}{lcc}
\hline Parameters & Lupeol & Lupenone \\
\hline Energy (in au) & -0.1855 & -0.1666 \\
HOMO (in eV) & -9.10 & -10.07 \\
LUMO (in eV) & 0.99 & 1.08 \\
Frontier orbital energy gap (in eV) & 10.09 & 11.15 \\
Dipole moment (in Debye) & 1.66 & 2.91 \\
\hline
\end{tabular}

The importance of MESP lies in the fact that it simultaneously displays molecular size, shape as well as positive, negative, and neutral electrostatic potential regions in terms of colour grading (shown in Figure 9) and is very useful in the investigation of molecular structure with its physiochemical property relationship [28-32]. The total dipole moment in the Cartesian frame is defined by

$$
\mu=\left(\mu_{x}^{2}+\mu_{y}^{2}+\mu_{z}^{2}\right)^{1 / 2}
$$

The calculated dipole moments for lupeol and lupenone are 1.66 and 2.91 debye, respectively. Comparison of the calculated dipole moment of given compounds with water show that it is more than water for lupenone and lower for lupeol. [A dipole moment value for water is 2.16 Debye, at the same level of calculations that is, B3LYP/6-31G: PM3]. 


\section{Conclusion}

In the present work we have calculated the geometric parameters, vibrational frequencies, frontier molecular orbitals, molecular electrostatic potential plots and surfaces for lupeol and lupenone using B3LYP/6-31G: PM3 method. The higher frontier orbital gaps show that lupeol and lupenone have high kinetic stability and can be termed as hard molecule. However the higher value of dipole moment shows that lupenone molecule is highly polar and for lupeol it is less polar. In general, a good agreement between experimental and calculated normal modes of vibrations has been observed. The molecular electrostatic potential plots and surfaces have also been drawn to explain the activity of both the molecules. The present quantum chemical study may further play an important role in understanding of the structure, activity and dynamics of the molecule.

\section{Acknowledgments}

The authors (N. Misra and A. Dwivedi) are grateful to the University Grants Commission, New Delhi for providing the financial assistance.

\section{References}

[1] A. Wal, P. Wal, A. K. Rai, and K. Raj, "Isolation and modification of pseudohybrid plant (Lupeol)," Journal of Pharmaceutical Sciences and Research, vol. 2, no. 1, pp. 13-25, 2010.

[2] S. Kumar, N. Misra, K. Raj, K. Srivastava, and S. K. Puri, "Novel class of hybrid natural products derived from lupeol as antimalarial agents," Natural Product Research, vol. 22, no. 4, pp. 305-319, 2008.

[3] S. Kumar, Chemical investigation of some important medicinal plants and chemical modification of phytochemicals, Ph.D. thesis, HNBGU, 2006.

[4] N. Misra, Study of natural products: isolation and synthesis of pseudohybrid natural products, Ph.D. thesis, Agra University, 2007.

[5] Selected Medicinal Plants of India, A Monograph of Identity, Safety, and Clinical Usage, CHEMEXCIL, 1992.

[6] T. Geetha and P. Varalakshmi, "Anti-inflammatory activity of lupeol and lupeol linoleate in rats," Journal of Ethnopharmacology, vol. 76, no. 1, pp. 77-80, 2001.

[7] M. J. Frisch et al., Gaussian, Inc., Wallingford, Conn, USA, 2009.

[8] S. Antonczak, G. Monard, M. F. Ruiz-López, and J. L. Rivail, "Modeling of peptide hydrolysis by thermolysin. A semiempirical and QM/MM study," Journal of the American Chemical Society, vol. 120, no. 34, pp. 8825-8833, 1998.

[9] R. K. Szilagyi, D. G. Musaev, and K. Morokuma, "Theoretical studies of biological nitrogen fixation-part II. Hydrogen bonded networks as possible reactant and product channels," Journal of Molecular Structure, vol. 506, pp. 131-146, 2000.

[10] S. Franzen, "Use of periodic boundary conditions to calculate accurate $\beta$-sheet frequencies using density functional theory," Journal of Physical Chemistry A, vol. 107, no. 46, pp. 9898-9902, 2003.

[11] M. V. Vener, A. N. Egorova, D. P. Fomin, and V. G. Tsirelson, "Hierarchy of the non-covalent interactions in the alanine-based secondary structures. dft study of the frequency shifts and electron-density features," Journal of Physical Organic Chemistry, vol. 22, no. 3, pp. 177-185, 2009. 
[12] R. A. Friesner and B. D. Dunietz, "Large-scale ab initio quantum chemical calculations on biological systems," Accounts of Chemical Research, vol. 34, no. 5, pp. 351-358, 2001.

[13] G. Mlinsek, M. Novic, M. Hodoscek, and T. Solmajer, "Prediction of enzyme binding: human thrombin inhibition study by quantum chemical and artificial intelligence methods based on X-ray structures," Journal of Chemical Information and Computer Sciences, vol. 41, no. 3-6, pp. 12861294, 2001.

[14] J. Nishihira and H. Tachikawa, "Theoretical evaluation of a model of the catalytic triads of serine and cysteine proteases by ab initio molecular orbital calculation," Journal of Theoretical Biology, vol. 196, no. 4, pp. 513-519, 1999.

[15] Y. H. Liang and F. E. Chen, "ONIOM DFT/PM3 calculations on the interaction between dapivirine and HIV-1 reverse transcriptase, a theoretical study," Drug Discoveries \& Therapeutics, vol. 1, no. 1, pp. 57-60, 2007.

[16] R. Wieczorek and J. J. Dannenberg, "Enthalpies of hydrogen-bonds in $\alpha$-helical peptides. An ONIOM DFT/AM1 study," Journal of the American Chemical Society, vol. 127, no. 42, pp. 14534 $14535,2005$.

[17] H. M. Senn and W. Thiel, "QM/MM methods for biomolecular systems," Angewandte Chemie, vol. 48, no. 7, pp. 1198-1229, 2009.

[18] J. Shiru, M. Zhiwen, D. Yujie, Z. Xiuli, Y. Hongjiang, and Q. Yuhua, "Computational study on the conformation and vibration frequencies of $\beta$-sheet of $\varepsilon$-polylysine in vacuum," International Journal of Molecular Sciences, vol. 10, no. 8, pp. 3358-3370, 2009.

[19] T. Matsubara, M. Dupuis, and M. Aida, "Ab initio ONIOM-Molecular Dynamics (MD) study on the deamination reaction by cytidine deaminase," Journal of Physical Chemistry B, vol. 111, no. 33, pp. 9965-9974, 2007.

[20] A. Frisch, A. B. Nelson, and A. J. Holder, Gauss View, Inc., Pittsburgh, Pa, USA, 2005.

[21] K.-C. Chou, "Biological functions of low-frequency vibrations (phonons) - III. Helical structures and microenvironment," Biophysical Journal, vol. 45, no. 5, pp. 881-889, 1984.

[22] H. Frohlich, Biological Coherence and Response to External Stimuli, Springer, Berlin, Germany, 1988.

[23] M. Alcolea Palafox, G. Tardajos, A. Guerrero-Martínez et al., "FT-IR, FT-Raman spectra, density functional computations of the vibrational spectra and molecular geometry of biomolecule 5aminouracil," Chemical Physics, vol. 340, no. 1-3, pp. 17-31, 2007.

[24] J. S. Singh, "FTIR and Raman spectra and fundamental frequencies of biomolecule: 5-Methyluracil (thymine)," Journal of Molecular Structure, vol. 876, pp. 127-133, 2008.

[25] C. P. Beetz Jr. and G. Ascarelli, "The low frequency vibrations of pyrimidine and purine bases," Spectrochimica Acta Part A, vol. 36, no. 3, pp. 299-313, 1980.

[26] J. Bandekar and G. Zundel, "The role of CO transition dipole-dipole coupling interaction in uracil," Spectrochimica Acta Part A, vol. 39, no. 4, pp. 337-341, 1983.

[27] I. Fleming, Frontier Orbitals and Organic Chemical Reactions, John Wiley and Sons, New York, NY, USA, 1976.

[28] J. S. Murray and K. Sen, Molecular Electrostatic Potentials, Concepts and Applications, Elsevier, Amsterdam, The Netherlands, 1996.

[29] I. Alkorta and J. J. Perez, "Molecular polarization potential maps of the nucleic acid bases," International Journal of Quantum Chemistry, vol. 57, no. 1, pp. 123-135, 1996.

[30] E. Scrocco and J. Tomasi, Advances in Quantum Chemistry, Edited by P. Lowdin, Academic Press, New York, NY, USA, 1978. 
[31] F. J. Luque, M. Orozco, P. K. Bhadane, and S. R. Gadre, "SCRF calculation of the effect of hydration on the topology of the molecular electrostatic potential," Journal of Physical Chemistry, vol. 97, no. 37, pp. 9380-9384, 1993.

[32] J. Šponer and P. Hobza, "DNA base amino groups and their role in molecular interactions: Ab initio and preliminary density functional theory calculations," International Journal of Quantum Chemistry, vol. 57, no. 5, pp. 959-970, 1996. 


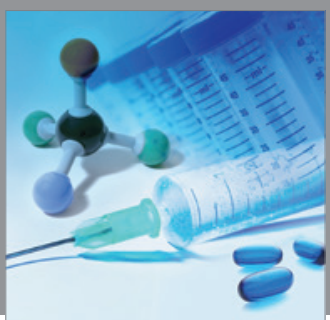

International Journal of

Medicinal Chemistry

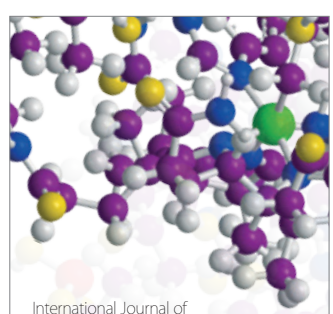

Carbohydrate Chemistry

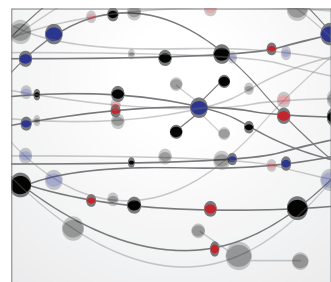

The Scientific World Journal
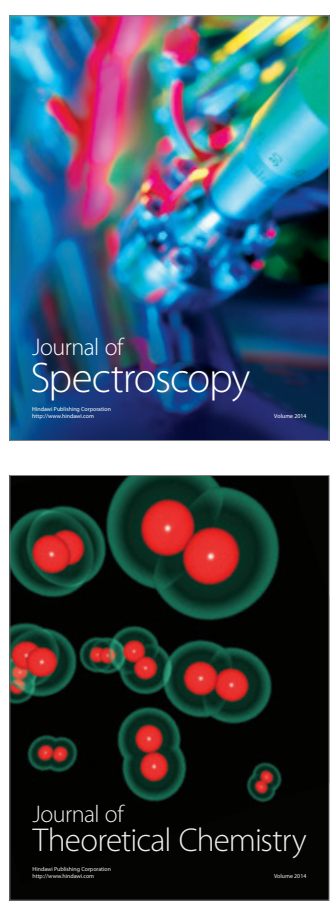
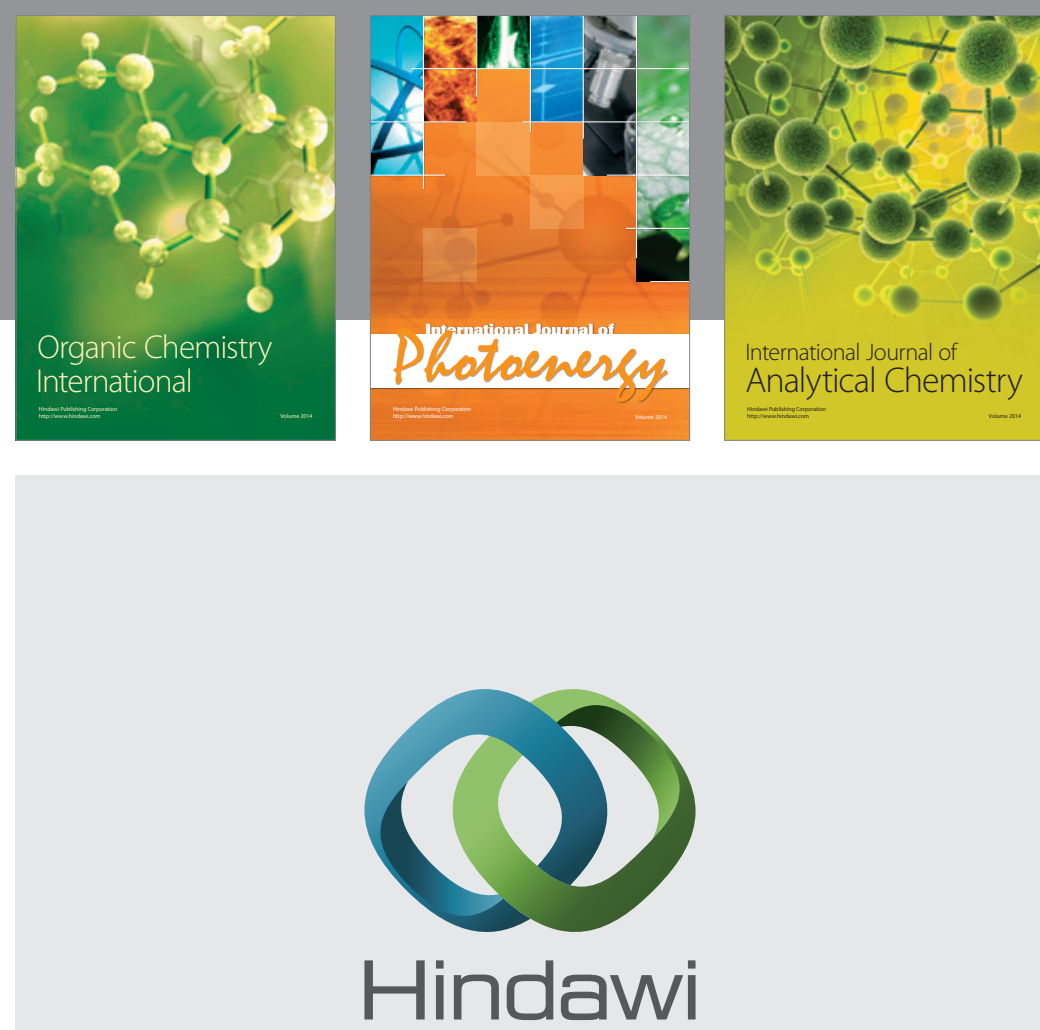

Submit your manuscripts at

http://www.hindawi.com
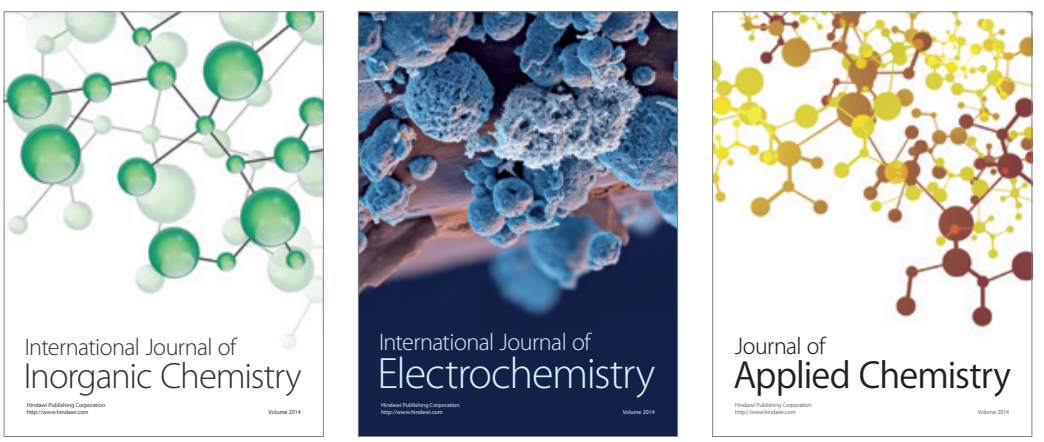

Journal of

Applied Chemistry
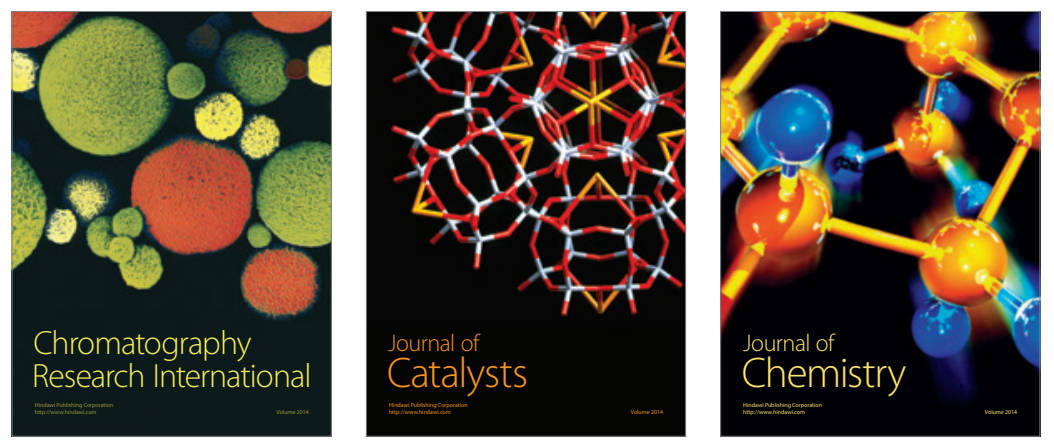
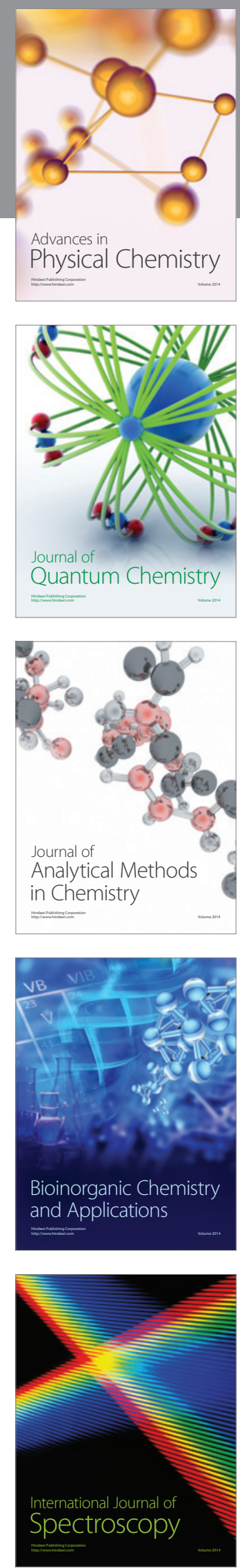